\title{
Isolation of biologically active compounds from mangrove sediments
}

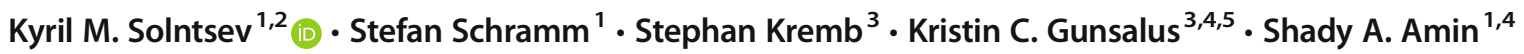 \\ Published online: 17 July 2019 \\ (C) Springer-Verlag GmbH Germany, part of Springer Nature 2019
}

The isolation of bioactive compounds from natural sources is a growing field, in particular due to its potential application in pharmacology and biotechnology. The relevance of this research was the driver to design a full-semester undergraduate analytical laboratory course to introduce students to advanced analytical and spectroscopic techniques while simultaneously exposing them to problems solved by modern research. The students extracted and characterized a biologically active compound from bacteria isolated from mangrove forest sediments. The bacterium, Vibrio sp. SKC6, was grown in a teaching laboratory setting and its intracellular metabolomic fraction obtained via liquid-liquid extraction, further purified by highperformance liquid chromatography (HPLC) and assayed for bioactivity using phenotypic profiling of a human cell line (HeLa). One of the HPLC fractions resulted in cell division arrest after DNA replication. The structure of the compound responsible for the observed bioactivity was characterized using mass spectrometry (UHPLC/ESI QToF-MS), ${ }^{1} \mathrm{H}$ and ${ }^{13} \mathrm{C}$ nuclear magnetic resonance (NMR) spectroscopy, and single-

Electronic supplementary material The online version of this article (https://doi.org/10.1007/s00216-019-02001-y) contains supplementary material, which is available to authorized users.

Kyril M. Solntsev

solntsev@gatech.edu

Shady A. Amin

samin@nyu.edu

1 Chemistry Division, New York University Abu Dhabi, PO Box 129188, Abu Dhabi, United Arab Emirates

2 School of Chemistry and Biochemistry, Georgia Institute of Technology, Atlanta, GA 30332-0400, USA

3 NYU Abu Dhabi Center for Genomics and Systems Biology, New York University Abu Dhabi, PO Box 129188, Abu Dhabi, United Arab Emirates

4 Biology Division, New York University Abu Dhabi, PO Box 129188, Abu Dhabi, United Arab Emirates

5 Center for Genomics and Systems Biology, Department of Biology, New York University, New York, NY, USA crystal X-ray diffraction analyses. This molecule was identified as pyridine-2,6-dicarboxylic acid, commonly known as dipicolinic acid (DPA), which is thought to be responsible for the thermotolerance of bacterial endospores. The findings from this course were summarized in a regular collaborative report written by each group of students. A unique, broad combination of analytical and biochemical methods makes this project attractive to students considering further careers in chemistry, chemical biology, and pharmaceutical drug discovery.

\section{Introduction}

Project-Based Learning has proven to be an effective teaching method in analytical chemistry [1, 2]. During a Project-Based Learning scheme, students are introduced to a combination of standard and state-of-the-art analytical techniques to emphasize an integrated and relevant approach to modern problems faced by chemists [3]. These projects involve utilization of various quantitative and qualitative analytical methods and often last from a few weeks [4] to a whole semester [1]. In the current paper, a combination of analytical and biochemical methods was applied to isolate and characterize biologically active natural products from mangrove forest sediments.

Natural products (NPs) are often chemical compounds found in nature, many of which appear to have evolved to efficiently interact with specific biological targets [5-8]. They have traditionally played a key role in drug discovery, with a significant fraction of medicines being derived from chemical modifications of the active components of plants commonly used in traditional medicines [8,9]; many others, including most known antibiotics, are derived from microbes (fungi and bacteria) $[10,11]$. In recent years, natural product discovery has experienced renewed interest stemming from the development of innovative techniques allowing for efficient bioactivity screening, isolation, and characterization of compounds, and the demand for novel antibacterial and antifungal compounds due to increasing resistance of pathogens, such as methicillinresistant Staphylococcus aureus (MRSA) [12]. Multiple factors 
have contributed to the development of the field including the large biodiversity of sources of natural compounds and costefficiency in comparison with their synthetic counterparts [11]. In fact, around $40 \%$ of chemical scaffolds in the Dictionary of Natural Products are a result of NP discovery and nearly $75 \%$ of approved anticancer drugs are NPs or derivatives of NPs [11, 13]. There is a growing interest in samples from marine organisms, particularly marine bacteria, that have been found to produce chemically novel bioactive metabolites [14-16]. This avenue of research is an exciting one given that oceans cover more than $70 \%$ of the earth's surface and an estimated $99 \%$ of all marine bacteria have not yet been cultured $[17,18]$. Since 2008, more than 1000 new chemical compounds have been discovered from marine organisms each year, the majority of which come from bacteria $[19,20]$. As culprits of infectious diseases continue to evolve and build resistance to available pharmaceuticals, NPs isolated from the marine environment provide a reservoir of potential cures against cancer, bacterial, fungal, parasitic, and viral diseases [21].

Mangrove forests represent a potential reservoir of untapped and unique NPs with a wide range of potential industrial applications. Mangrove sediments are extreme environments that exhibit high salinity and temperature, low oxygen concentrations, strong UV exposure, and high density of microbes due to strong organic nutrient input from mangrove roots. This is especially true for the site of collection in this course, the Arabian Gulf near the shores of Abu Dhabi, UAE [22]. Such an extreme environment may force microbes to compete with one another by producing novel NPs, some of which could be developed as new antibiotics or antifungals. Compounds present in other mangrove species also exhibit interesting biological activities. Examples include halophytederived antioxidants that show little or no cytotoxicity (desirable characteristics in the food and cosmetic industries) [23] and the marine alkaloid ecteinascidin-743 (Trabectedin), isolated from the mangrove tunicate Ecteinascidia turbinata, which exhibits promising activity against melanomas and numerous carcinomas [24]. This drug is approved by the FDA and European Commission and is manufactured under the commercial name of Yondelis [25]. In this context, an analytical laboratory course was developed to expose students to modern analytical and spectroscopic techniques combined with useful microbiological and pharmaceutical applications.

\section{Methods}

The goal of the project is to expose students to the isolation and characterization of secondary metabolites from marine bacteria. The project consists of 2-3 groups of students working to grow a bacterium isolated from mangrove sediments and then extract, isolate, and characterize the structure of one unknown molecule (to the students) that displays potentially interesting bioactivity in HeLa cells. All participants were divided into teams of 2-3 junior or senior undergraduate students majoring in chemistry at New York University Abu Dhabi and New York University Shanghai. Implementation of the laboratory took place during the first semester of three consecutive years (2015-2017). The laboratory sessions met 240 min per week for 14 weeks. It is important to mention that the laboratory course described in this paper is a component of the overall Analytical Chemistry course which includes weekly lectures. The syllabus was arranged in a way to introduce the analytical techniques in the lectures prior to their appearance in the laboratory. In this report, one secondary metabolite was isolated by two groups from Vibrio sp. SKC6 (SKC6, accession no. MH179312) as discussed. Three other secondary metabolites were isolated by other groups from SKC6 but are not discussed because the structures of these compounds could not be determined or were partially determined.

The process (Fig. 1) starts with each group growing in liquid culture a marine bacterium, SKC6, previously isolated from mangrove sediments. SKC6, a strain closely related to a suspected crustacean pathogen (Vibrio owensii) [26], was chosen because it readily grew in liquid medium and produced a bioactive compound that exhibited toxicity against HeLa cells, both of which were ideal characteristics for a natural product project. Students grow their bacterium to stationary phase and isolate organic biomolecules from cell pellets using liquidliquid extraction to acquire a crude bacterial "extract" that is further separated on a high-performance liquid chromatography (HPLC) instrument. Small aliquots from the HPLC fractions are tested separately by the instructor(s) for decreased cell survival (cytotoxicity) and other biological parameters against HeLa cells. Students further purify the bioactive fraction using HPLC chromatography to acquire a pure fraction. Using mass spectrometry, NMR spectroscopy, and X-ray diffraction (Fig. 1), the students characterize the structure of their unknown. The entire project conducted by each group throughout the semester is summarized in a written collaborative report prepared by each group (Supporting Information). Detailed experimental procedures are included in the Supporting Information.

\section{Hazards}

All students passed the following safety training courses required by NYU Abu Dhabi: Laboratory Safety Induction, Biosafety Induction, Electrical Safety in the Laboratory, Use of Laboratory Hoods, Laboratory Emergencies. Personal protection equipment (goggles, lab coats, gloves, closed-toe shoes) must be worn when working with hazardous and flammable reagents. Methanol is flammable; methanol, acetonitrile, and dichloromethane are hazardous to humans and the 


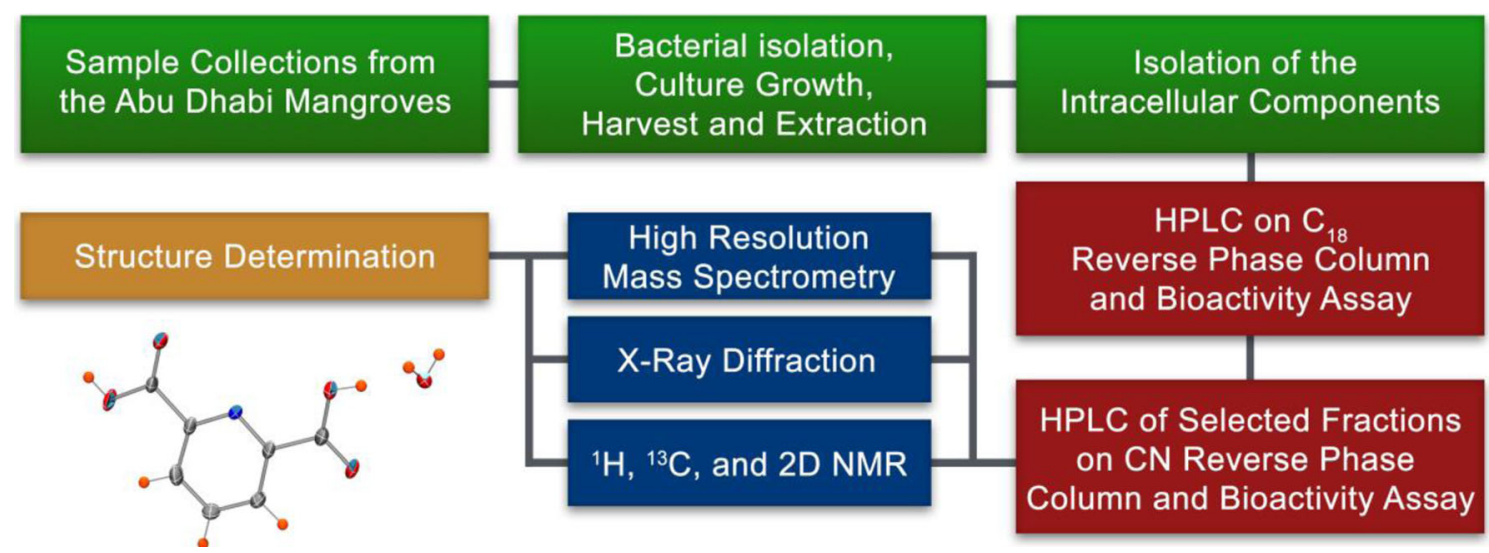

Fig. 1 A work flowchart detailing the steps of the method to isolate biologically active compounds from the bacterial culture collected from mangrove sediments

environment and must be handled with care and disposed of properly. Trifluoroacetic acid (TFA) is corrosive.

\section{Results and discussion}

The first purification of crude extracts of the intracellular fractions of the bacterium SKC6 on a reversed phase $\mathrm{C}_{18}$ column using a general, wide gradient showed the presence of many biomolecules as evidenced by UV absorption at $220 \mathrm{~nm}$ (Fig. 2a). The bioassay exposed HeLa cells to HPLC fractions and monitored effects on mitotic index and the shape, intensity, and size of the nucleus, actin, mitochondria, and lysosomes [27]. Bioactive fractions (Fig. S1) showed an increase in the proportion of cells with $4 \mathrm{~N}$ vs. $2 \mathrm{~N}$ chromosome complements, indicating cell cycle arrest after DNA replication. The fractions eluting between 3 and 5 min showed moderate cytotoxicity and caused significant changes to several cellular characteristics (Fig. S1).

Since the bioactive fraction eluted at low concentrations of $\mathrm{CH}_{3} \mathrm{CN}$, students reasoned that it consisted of polar molecules. Thus, the active fraction was further purified using a gradient with small increments of $\mathrm{CH}_{3} \mathrm{CN}$ concentrations on the same $\mathrm{C}_{18}$ column. The fractions eluting at 11-13 min showed strong bioactivity on HeLa cells (Fig. S1). The HPLC chromatogram obtained indicated a sample with several molecules (Fig. S2). Crystals were observed in these fractions, indicating that the sample was relatively pure. To further purify the bioactive fractions, students used HPLC and a CN column to increase the separation of polar compounds from the second purification, which resulted in a pure fraction of the bioactive molecule (Fig. 2a). The solvent gradient and composition were optimized by students to achieve the optimal separation. UHPLC-QToF mass spectrometry analysis indicated a molecular ion peak $[\mathrm{M}+\mathrm{H}]^{+}$at $\mathrm{m} / \mathrm{z} 168.0285$, with an $\mathrm{M}+1$ peak at $7.04 \%$ relative intensity (Fig. 2b). The a

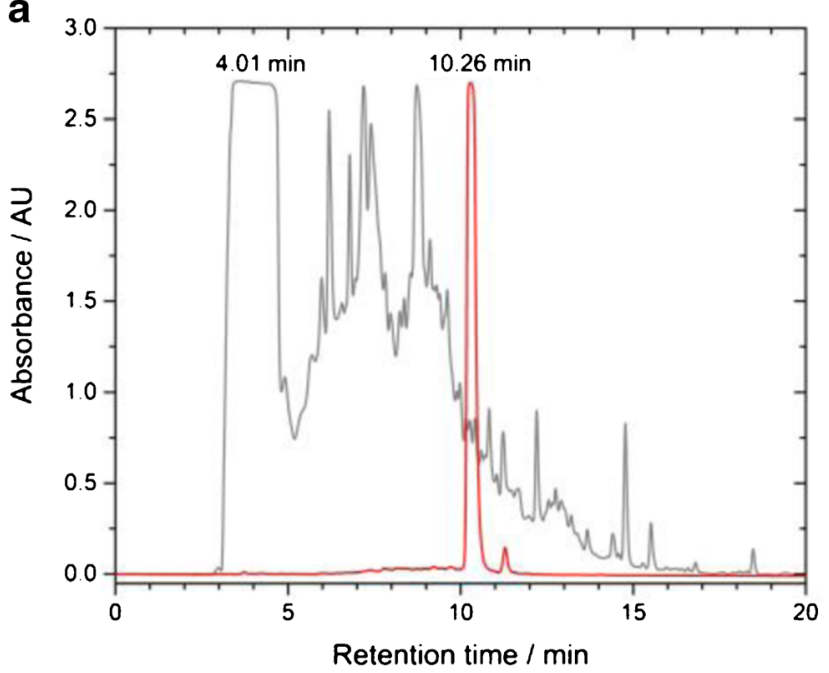

b

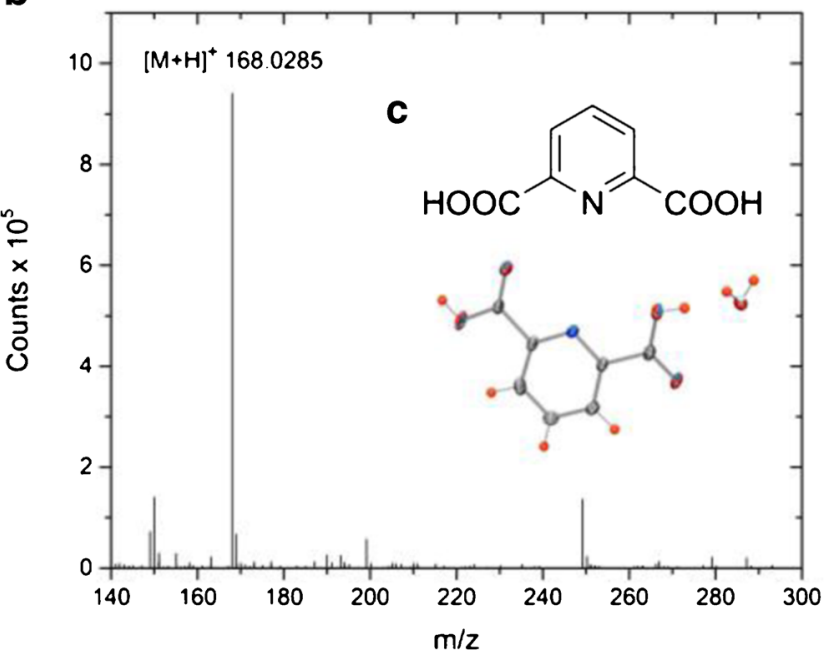

Fig. 2 a HPLC chromatogram measured with absorbance at $220 \mathrm{~nm}$ of the first crude HPLC purification on $\mathrm{C}_{18}$ (gray) and final purification on $\mathrm{CN}$ (red) columns. b High-resolution UHPLC-QToF mass spectrum of the purified sample of dipicolinic acid (DPA), showing a prominent $\mathrm{M}+$ $\mathrm{H}]^{+}$peak at $\mathrm{m} / \mathrm{z}$ of 168.0285 . c Structure and ORTEP diagram of DPA drawn with $50 \%$ ellipsoidal probability 
predominant molecular ion was searched by students on the Scripps Center for Metabolomics database (METLIN: metlin.scripps.edu) with an error tolerance of $\pm 5 \mathrm{ppm}$. This search produced four metabolites with an identical molecular formula, $\mathrm{C}_{7} \mathrm{H}_{5} \mathrm{NO}_{4}$ : 2,3-pyridinedicarboxylic acid, dipicolinic acid, and $p$ - and $o$-nitrobenzoic acid.

The pure fraction dissolved in methanol- $d_{4}$ was analyzed using NMR spectroscopy. In a students' sample ${ }^{1} \mathrm{H}-\mathrm{NMR}$ spectrum, two peaks $(d, 8.37 \mathrm{ppm} ; t, 8.22 \mathrm{ppm})$ in the aromatic region indicated the potential presence of a symmetrical compound (Figs. 3 and S3). The ${ }^{13} \mathrm{C}-\mathrm{NMR}$ spectrum showed four non-equivalent carbons (Fig. S4), again indicating the presence of high symmetry in this compound given the molecular formula. The HSQC NMR spectrum, along with the ${ }^{1} \mathrm{H}$ and ${ }^{13} \mathrm{C}$ spectra, unequivocally confirmed that the purified molecule is dipicolinic acid, DPA (Fig. 4). A full UV-Vis spectrum was collected as well at this point for DPA, which shows maximum absorbance at $\sim 275 \mathrm{~nm}$ (Fig. 5).

Because crystals were observed after drying semi-pure fractions from the SpeedVac, the structure of the purified compound was solved by X-ray diffraction (Fig. 2c, Table S1). The refined

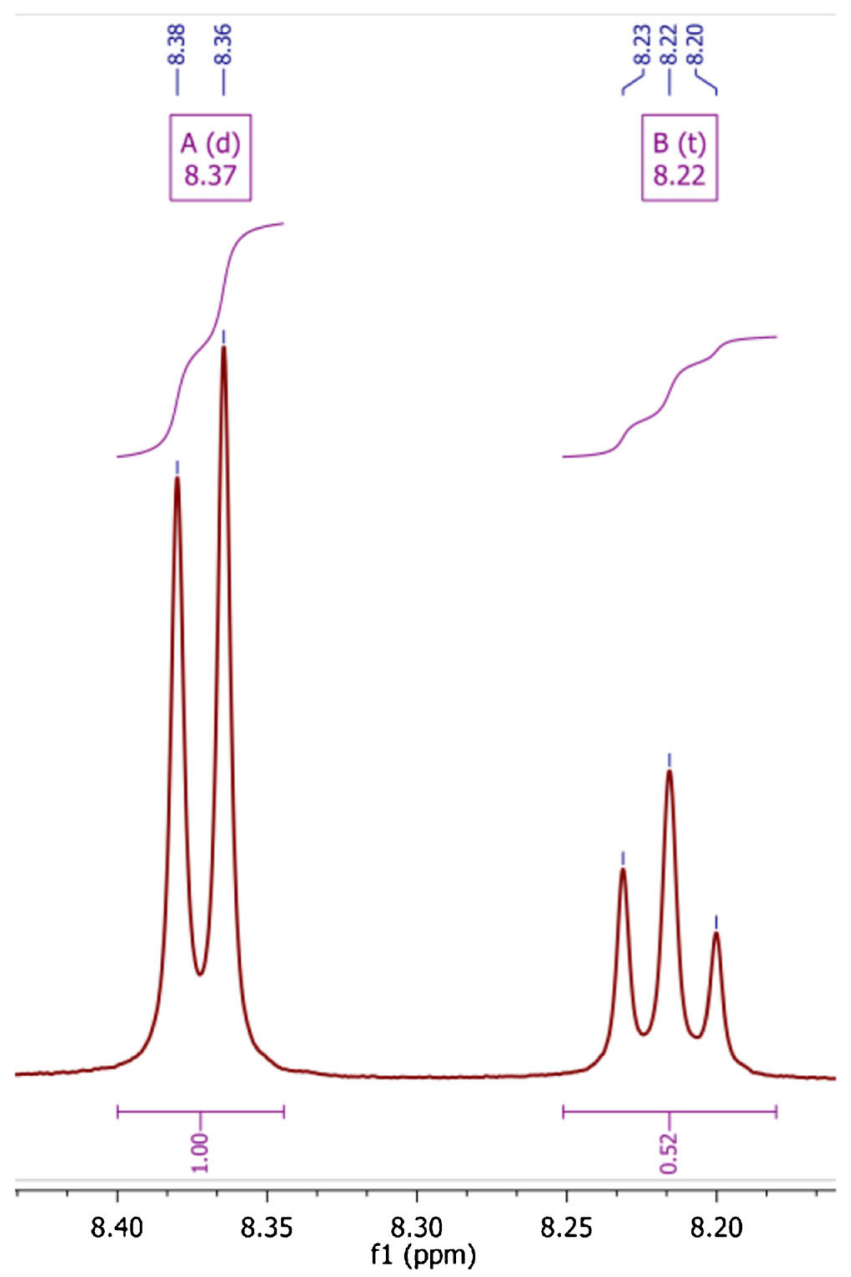

Fig. $3{ }^{1} \mathrm{H}$ NMR spectrum of dipicolinic acid. Only the aromatic part is shown. For the full spectra including solvent residual peaks, see Fig. S3 structure confirms mass spectrometry and NMR spectroscopy findings and definitively identifies the molecule as DPA.

The course was designed to expose students to hands-on experience in running instruments and analyzing output data as realistically possible. Because the project relied on several state-of-the-art research instruments, the ability of students to directly operate some of these instruments was limited. For example, XRD operation and data analysis were completely conducted by a crystallography research scientist. However, the XRD structure was only provided to students after DPA was characterized via NMR spectroscopy. For mass spectrometry, students set up their experiments with the help of the instructor. Students directly operated the NMR instrument. Data interpretation for both techniques was done solely by the students.

The semester was concluded for this laboratory by taking the students on a field expedition to nearby mangroves, where the students collected samples for bacterial isolation and recorded environmental parameters. This exercise helped the students visualize and experience how their bacterial samples were collected, providing context and greater appreciation for the full development cycle of this project. All the findings reported here were summarized by the students in a collaborative report in the ACS-style, a part of which is included in the "Supplementary Information" section. The progress of students was assessed mainly by reviewing an initial draft of their report two weeks prior to the final draft's due date. Evaluation of the students' writing skills, chemistry knowledge, ability to review and summarize scientific literature, and ability to accurately describe and interpret their findings was conducted by the instructor at this phase.

Natural product isolation and characterization has been an intensive area of pedagogical activity covered by current literature. Many previously published laboratory experiments pertaining to the subject include one or more standard steps: extraction of the NPs from crude material, separation and analysis using various chromatographic techniques, identification and characterization of the isolated compounds, and determination of their biological activity. Similar to work presented here, extraction procedures involved the utilization of various organic solvents [28-30]. The range of chromatographic techniques varied from simple paper chromatography [31] and TLC [30, 32-34], with the latter noting, "the students recognize that TLC does not have the resolving power necessary to separate the extracted compounds and are guided towards a more powerful alternative, HPLC" [35]. HPLC is widely used in undergraduate teaching environments [35-38], so the utilization of HPLC in this paper is an obvious choice given the complexity of the extract. For characterization of compounds, gas chromatography, sometimes coupled to a mass spectrometer (GC-MS), has been utilized [29, 39, $40]$ and in some cases, high-resolution mass spectrometry has also been used [39]. Most of the papers referenced above used 
Fig. 4 HSQC NMR spectrum of dipicolinic acid

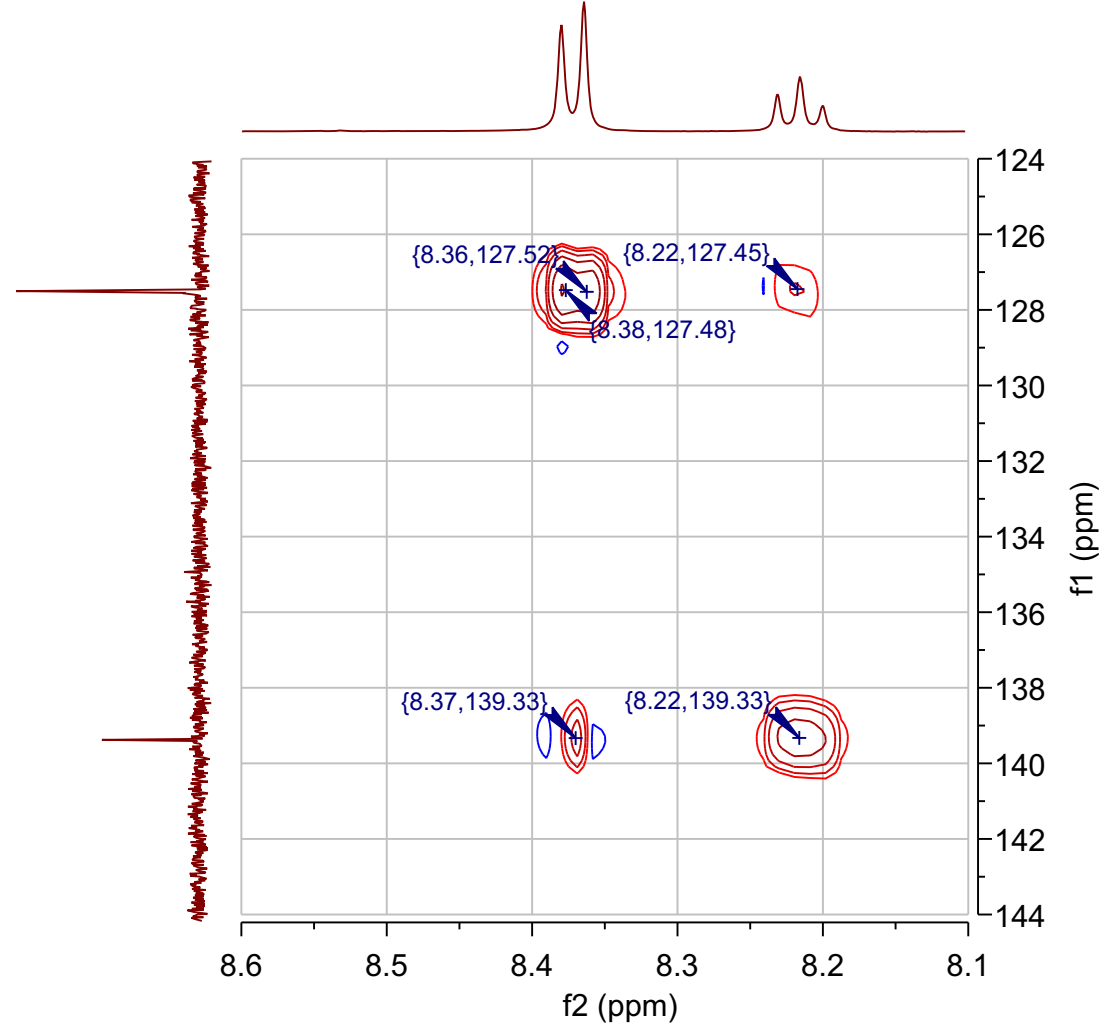

demonstrate that the cell assays presented here are in line with previous laboratory experiments conducted with undergraduate students. The advantage of the work described here is that it can be modified and manipulated to isolate different molecules from different environmental sources. Some alternatives to the environment and the assays and techniques reported here are listed below and in the Supporting Information (Instructions for Instructors). In addition, the bioassay reported here utilizes a novel phenotypic profiling of HeLa cells. This technique combines the use of several fluorescent markers to measure a variety of cellular functions in a high-
Fig. 5 UV-Vis absorption spectrum of DPA in 50:50 v/v methanol/water solution

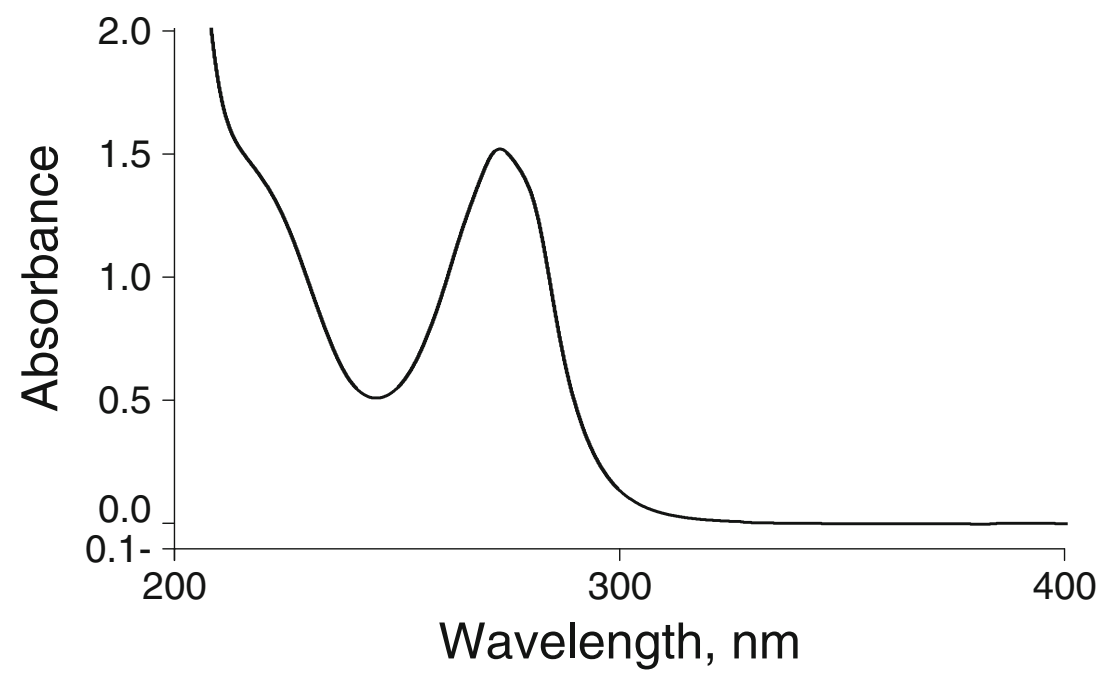


throughput manner, which provides a wealth of information on the cellular response to natural products [43, 44]. Besides its novelty, such a technique will expose chemistry students to cutting-edge multidisciplinary research.

This course was designed to use a bacterium isolated previously from mangrove sediments, an environment not universally accessible to many educators. Bacterial isolation from other sources (e.g., lakes, seawater, ponds) can be achieved by simple early modification of the procedures while keeping the majority of downstream purification intact. The Supporting Information (Instructions for Instructors) is amended with a paragraph on how to diversify bacterial isolation and growth from non-mangrove environments. Obviously, the separation and isolation of other metabolites from other sources will require a different HPLC protocol, but the Instructions submitted in Supplementary Information could be a good starting point.

A major hurdle that some educators may face is the inability to grow large volumes of bacteria $(>10 \mathrm{~L})$ due to limited incubator-shaker equipment. Although students in this course grew $12 \mathrm{~L}$ of SKC6 to achieve $>30 \mathrm{mg}$ of DPA, the amount needed for mass spectrometry and NMR spectroscopy is significantly less (likely $1-3 \mathrm{mg}$ ). Therefore, reducing the volume by half or more will theoretically still yield copious amounts of DPA. Supporting Information (Instructions for Instructors) now contains information about reducing the volume of bacterial cultures to simplify the workflow for laboratories that cannot grow large volumes.

Another hurdle for some educators is the use of tissue culture facilities to examine the biological activities of extracts and purified fractions. Although the instructor and students acquired data using a high-content screening platform, similar results can be obtained using a basic proliferation assay, which relies on measuring the mitochondrial enzymes of a cell by simple colorimetric measurements [45]. Using a simple plate reader, screening of 96 fractions or more would involve minimal efforts and still detect cytotoxicity of DPA. Alternatively, fixing cells, staining them with a DNA fluorescent dye, such as SYBR Green, and quantifying the DNA using fluorescence could also be used in lieu of high-content screening. Another alternative is to conduct a simple cell count of treated cells relative to controls that would also yield information about cytotoxicity and could be performed by students within the context of the course.

The availability of high-resolution mass spectrometry instruments, such as LC-QToF-MS used here, may be limited in some institutions. In lieu of such instruments, a simple gas chromatography (GC) system or GC-MS can still be used to characterize DPA. Supporting Information (Fig. S5) now contains a GC-MS analysis of DPA in case high-resolution MS is not.

Finally, we note that the isolation of the natural products is not an easy task. Several times during the course, the concentration of the isolated molecules was so low and/or the structure was complex enough that the target molecules were not fully characterized. Nevertheless, the students actually found it exciting that they were perhaps onto something new, and this offered them the opportunity to engage creatively in the research experience. In addition, previously published J. Chem. Ed. Laboratory Experiments dealing with natural product isolation also reported students partially characterizing molecules or completely failing to do so [42].

\section{Summary}

Throughout the semester, students gained hands-on experience working in small groups to address a challenging, yet relevant, problem in chemistry: the discovery and characterization of natural products. The pedagogic goal of this exercise was to expose students to a semester-long continuous experiment that exposed them to state-of-theart methods in NP discovery and structure characterization. For example, the techniques and instruments learned by students during this laboratory are used in almost all chemical laboratories, giving the young scholars a rare glimpse into modern research. The project as a whole was designed to be completed in a 14-week semester by the students meeting $4 \mathrm{~h}$ per week. While direct involvement of the instructor was necessary to facilitate some of the tasks throughout the semester (e.g., bacterial growth, bioassays), the bulk of the work was carried out by students. To ensure feasibility, the laboratory required some validation prior to having the students conduct experiments: bacterial isolation, optimization of culture growth conditions, and testing of crude extracts from multiple samples to screen for bioactivity were all performed in advance. However, all HPLC purification, spectroscopy, and identification of the DPA were completely carried out by the students (except for maintenance of the mass spectrometer, NMR spectroscopy, and XRD). This exercise was successfully repeated during three separate semesters with different groups of students. In addition, the field excursion provided the students with a valuable experience in the importance of meticulous field sampling. Finally, the end-of-semester laboratory report that students wrote to summarize their findings helped them to consolidate their knowledge, practice scientific writing, and increase the collaborative skills that are essential in today's scientific world.

Acknowledgments We are indebted to all NYUAD undergraduate students who participated in this work. The help of Amin and Gunsalus laboratory members and NYUAD Core facilities personnel is highly appreciated. Parts of this research were carried out on the Core Lab facilities of New York University Abu Dhabi. We thank Durga Prasad Karothu for help with X-ray diffraction measurements. 
Funding information The field trip to the mangrove forest was made possible through a grant from the NYUAD Community-Based Learning Activities Center.

\section{References}

1. Davis EJ, Pauls S, Dick J. Project-Based Learning in undergraduate environmental chemistry laboratory: using EPA methods to guide student method development for pesticide quantitation. J Chem Educ. 2017;94:451-7.

2. Luna Vera F, Chalapud MG, Viveros IG, Medina EAV. From eggshells to quicklime: using carbonate cycle as an integrating concept to introduce students to materials analysis by TGA and FTIR. J Chem Educ. 2018;95:625-30.

3. Riley SRR. An integrated approach to thermal analysis of pharmaceutical solids. J Chem Educ. 2015;92:932-5.

4. Heider EC, Valenti D, Long RL, Garbou A, Rex M, Harper JK. Quantifying sucralose in a water-treatment wetlands: servicelearning in the analytical chemistry laboratory. J Chem Educ. 2018;95:535-42.

5. Haefner B. Drugs from the deep: marine natural products as drug candidates. Drug Discov Today. 2003;8:536-44.

6. Butler MS. The role of natural product chemistry in drug discovery. J Nat Prod. 2004;67:2141-53.

7. Koehn FE, Carter GT. The evolving role of natural products in drug discovery. Nat Rev Drug Discov. 2005;4:206-20.

8. Lam KS. New aspects of natural products in drug discovery. Trends Microbiol. 2007;15:279-89.

9. Newman DJ, Cragg GM, Snader KM. The influence of natural products upon drug discovery. Nat Prod Rep. 2000;17:215-34.

10. Clardy J, Fischbach M, Walsh C. New antibiotics from bacterial natural products. Nat Biotechnol. 2006;24:1541-50.

11. Strobel G, Daisy B, Castillo U, Harper J. Natural products from endophytic microorganisms. J Nat Prod. 2004;67:257-68.

12. Newman DJ, Cragg GM. Natural products as sources of new drugs over the 30 years from 1981 to 2010. J Nat Prod. 2012;75:311-35.

13. Montaser R, Luesch H. Marine natural products: a new wave of drugs? Future Med Chem. 2011;3:1475-89.

14. Harvey AL. Natural products in drug discovery. Drug Discov Today. 2008;13:894-901.

15. Burja AM, Banaigs B, Abou-Mansour E, Burgess JG, Wright PC. Secondary metabolites produced by the tropical marine filamentous cyanobacterium, Lyngbya majuscula - a prolific source of marine natural products. Tetrahedron. 2001;57:9347-77.

16. König GM, Kehraus S, Seibert SF, Abdel-Lateff A, Müller D. Natural products from marine organisms and their associated microbes. ChemBioChem. 2006;7:229-38.

17. Jimeno JM. A clinical armamentarium of marine-derived anti-cancer compounds. Anti-Cancer Drugs. 2002;13:S15-9.

18. Rappé MS, Giovannoni SJ. The uncultured microbial majority. Annu Rev Microbiol. 2003;57:369-94.

19. Hu Y, Chen J, Hu G, Yu J, Zhu X, Lin Y, et al. Statistical research on the bioactivity of new marine natural products discovered during the 28 years from 1985 to 2012. Mar Drugs. 2015;13:202-21.

20. Gerwick WH, Moore BS. Lessons from the past and charting the future of marine natural products drug discovery and chemical biology. Chem Biol. 2012;19:85-98.

21. Donia M, Hamann MT. Marine natural products and their potential applications as anti-infective agents. Lancet Infect Dis. 2003;3: $338-48$.

22. Sharqawy MH, Lienhard JH, Zubair SM. Thermophysical properties of seawater: a review of existing correlations and data. Desalin Water Treat. 2010;16:354-80.
23. Lopes A, Rodrigues MJ, Pereira C, Oliveira M, Barreira L, Varela J, et al. Natural products from extreme marine environments: searching for potential industrial uses within extremophile plants. Ind Crop Prod. 2016;94:299-307.

24. Cvetkovic RS, Figgitt DP, Plosker GL. ET-743. Drugs. 2002;62: 1185-92.

25. Cuevas C, Francesch A. Development of Yondelis (trabectedin, ET743). A semisynthetic process solves the supply problema. Nat Prod Rep. 2009;26:322-37.

26. Cano-Gómez A, Goulden EF, Owens L, Høj L. Vibrio owensii sp. nov., isolated from cultured crustaceans in Australia. FEMS Microbiol Lett. 2010;302:175-81.

27. Gribble GW. Natural organohalogens: a new frontier for medicinal agents? J Chem Educ. 2004;81:1441-9.

28. Nazri MM, Samat FD, Kavanagh PV, Walsh JJ. Nature's cholesterol-lowering drug: isolation and structure elucidation of lovastatin from red yeast rice-containing dietary supplements. J Chem Educ. 2012;89:138-40.

29. Buckley HL, Beck AR, Mulvihill MJ, Douskey MC. Fitting it all in: adapting a green chemistry extraction experiment for inclusion in an undergraduate analytical laboratory. J Chem Educ. 2013;90:771-4.

30. Chrea B, O'Connell JA, Silkstone-Carter O, O'Brien J, Walsh JJ. Nature's antidepressant for mild to moderate depression: isolation and spectral characterization of hyperforin from a standardized extract of St. John's wort (Hypericum perforatum). J Chem Educ. 2014;91:440-2.

31. Galloway KR, Bretz SL, Novak M. Paper chromatography and UV -Vis spectroscopy to characterize anthocyanins and investigate antioxidant properties in the organic teaching laboratory. J Chem Educ. 2015;92:183-8.

32. McLain KA, Miller KA, Collins WR. Introducing organic chemistry students to natural product isolation. Using steam distillation and liquid phase extraction of thymol, camphor, and citral, monoterpenes sharing a unified biosynthetic precursor. J Chem Educ. 2015;92:1226-8.

33. Locock K, Bakas T, Sanai F, Allan R, Hinton T. What is the "Areca" in "Areca nuts"? Extraction and neuroactive bioassay of arecoline. J Chem Educ. 2016;93:197-201.

34. Fagundes TSF, Dutra KDB, Ribeiro CMR, Epifanio RA, Valverde AL. Using a sequence of experiments with turmeric pigments from food to teach extraction, distillation, and thin-layer chromatography to introductory organic chemistry students. J Chem Educ. 2016;93: 326-9.

35. Purcell SC, Pande P, Lin Y, Rivera EJ, Paw UL, Smallwood LM, et al. Extraction and antibacterial properties of thyme leaf extracts: authentic practice of green chemistry. J Chem Educ. 2016;93:1422-7.

36. Barrocas Dias C, Miranda M, Manhita A, Candeias A, Ferreira T, Teixeira D. Identification of onion dye chromophores in the dye bath and dyed wool by HPLC-DAD: an educational approach. J Chem Educ. 2013;90:1498-500.

37. Mann FM. Identification and analysis of bioactive components of fruit and vegetable products. J Chem Educ. 2015;92:892-5.

38. Halepas S, Hamchand R, Lindeyer SED, Brückner C. Isolation of biliverdin IX $\alpha$, as its dimethyl ester, from emu eggshells. J Chem Educ. 2017;94:1533-7.

39. Bendinskas K, Weber B, Nsouli T, Nguyen HV, Joyce C, Niri V, et al. A teaching laboratory for comprehensive lipid characterization from food samples. J Chem Educ. 2014;91:1697-701.

40. Just J, Bunton GL, Deans BJ, Murray NL, Bissember AC, Smith JA. Extraction of eugenol from cloves using an unmodified household espresso machine: an alternative to traditional steam-distillation. J Chem Educ. 2016;93:213-6.

41. Chinchilla N, Durán AG, Carrera C, Ayuso J, Macías FA. Operation allelopathy: an experiment investigating an alternative to synthetic agrochemicals. J Chem Educ. 2014;91:570-4. 
42. Hakim A, Liliasari, Kadarohman A, Syah YM. Making a natural product chemistry course meaningful with a mini project laboratory. J Chem Educ. 2016;93:193-6.

43. Caicedo JC, Singh S, Carpenter AE. Applications in image-based profiling of perturbations. Curr Opin Biotechnol. 2016;39:134-42.

44. Schulze CJ, Bray WM, Woerhmann MH, Stuart J, Lokey RS, Linington RG. 'Function-first' lead discovery: mode of action profiling of natural product libraries using image-based screening. Chem Biol. 2013;20:285-95.

45. Mosmann T. Rapid colorimetric assay for cellular growth and survival: applications to proliferation and cytotoxicity assays. J Immunol Methods. 1983;65:55-63.

Publisher's note Springer Nature remains neutral with regard to jurisdictional claims in published maps and institutional affiliations.

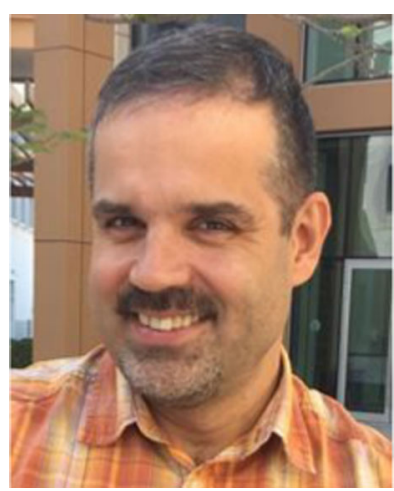

Kyril M. Solntsev received his $\mathrm{PhD}$ at Moscow State University working with Michael Kuz'min on excited-state proton transfer in various microheterogeneous systems. After a joint postdoctoral stint in Israel with Noam Agmon and Dan Huppert in the field of photoinduced processes in novel photoacids, Kyril moved to Georgia Tech. He was first a postdoctoral fellow with Laren Tolbert, then a research scientist and, finally, an independent PI at the School of Chemistry and Biochemistry. He has coauthored more than 90 papers in the areas of photochemistry, spectroscopy, chemical kinetics, fluorescent probes, and function and design of fluorescent proteins. A few years ago Kyril joined OLIS, Inc., an instrumental company in Bogart, GA, and then Ereztech, LLC in Atlanta, GA, as a senior staff scientist. Both companies are very science-oriented, and he actively continues research in various aspects of modern spectroscopy and photochemistry. He joint NYUAD in 2017 to teach Foundation of Science and Analytical Chemistry.

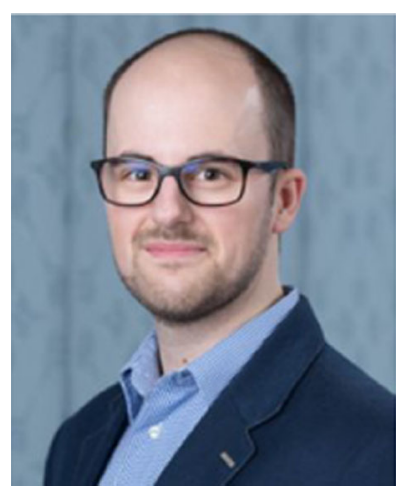

Stefan Schramm received his B.S. (2012) in chemistry and an M.S. (2014) in chemical biology from the Friedrich-SchillerUniversity Jena, Germany. He earned his Dr. rer. nat. in 2016 in chemistry from the FriedrichSchiller-University, Jena, Germany, in the group of Prof. R. Beckert by studying the chemiluminescence of 2-coumaranones with a special focus on synthesis, luminescence mechanism elucidation, and development of potential applications. From 2016-2018, he was a member of the group of Pancě Naumov at New York University Abu Dhabi as a Postdoctoral Associate. His research interests include the synthesis of small organic molecules, characterization of luminescent and advanced materials, and quantum chemical calculations in order to clarify reaction mechanisms and modeling of excited state geometries. He is a recipient of the FriedrichEbert Scholarship, the Marlene DeLuca Award 2016, and the A.R. Katritzky Prize 2017.

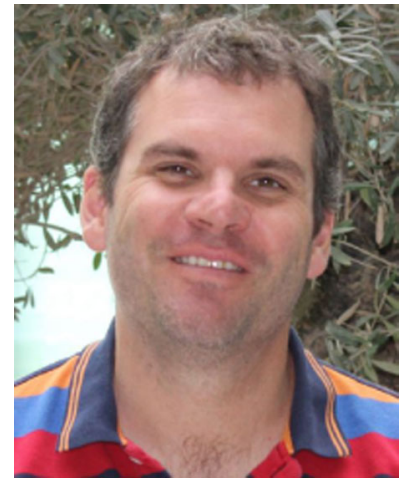

Stephan Kremb studied biology in Germany at the Technical University of Munich (TUM) and University of Bremen where he received his diploma in 2001 in the field of virology. His Ph.D. focused on UV-induced changes of gene expression in marine macroalgae. After receiving his Ph.D. from TUM in 2006, he joined the research group of Ruth Brack-Werner at the Institute of Virology of the Helmholz Center Munich where he worked on the identification of small-molecule inhibitors of the human immunodeficiency virus (HIV-1). From 2012-2015 he worked in the research group of Christian Voolstra at King Abdullah University of Science and Technology (KAUST) in Saudi Arabia where he established a high-content screening platform for the phenotypic profiling of natural products on a single cell level. $\mathrm{He}$ is currently a member of the Chemical and Functional Genomics group of Kristin Gunsalus at New York University Abu Dhabi where he is working on in-depth high-content phenotypic profiling of small molecules and bacterial natural products from the Arabian Gulf. 


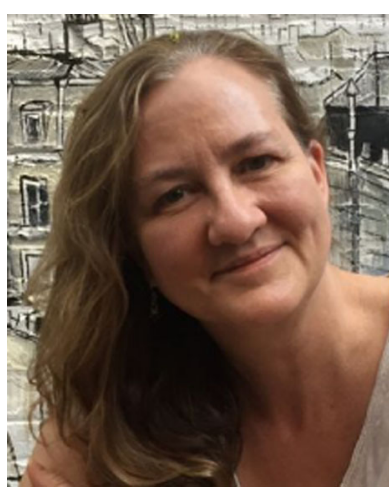

Kristin C. Gunsalus is Professor of Biology at NYU in New York and Affiliate Faculty at NYU Abu Dhabi (NYUAD), where she serves as Faculty Director of Bioinformatics and Co-Director of the NYUAD Center for Genomics and Systems Biology. She holds a B.A. in chemistry and biology and a Ph.D. in molecular genetics from Cornell University. She was a postdoctoral associate in structural genomics at Rutgers University and then moved into functional genomics and bioinformatics as a postdoctoral fellow at Cornell before moving to NYU in 2002. Her research spans a broad range of topics, including molecular genetic networks and evolution of early animal development, post-transcriptional regulation, and natural products discovery.

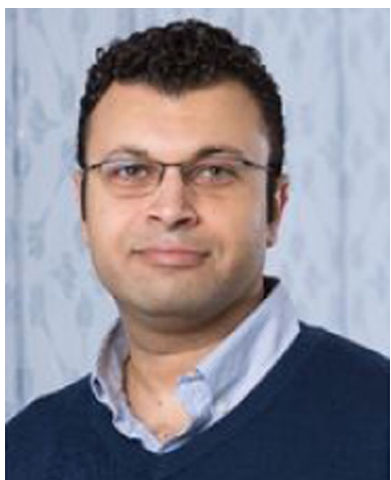

Shady A. Amin is a microbial chemical ecologist studying interspecies interactions of marine microbes. He received his B.Sc. in biochemistry from the University of California Santa Barbara, his $\mathrm{Ph} . \mathrm{D}$. in inorganic chemistry from the University of California San Diego and San Diego State University and continued for his postdoctoral work at the University of Washington's School of Oceanography. He joined New York University Abu Dhabi's Biology faculty in 2015, where his laboratory studies interactions between phytoplankton and bacteria and between corals and their microbiomes. 